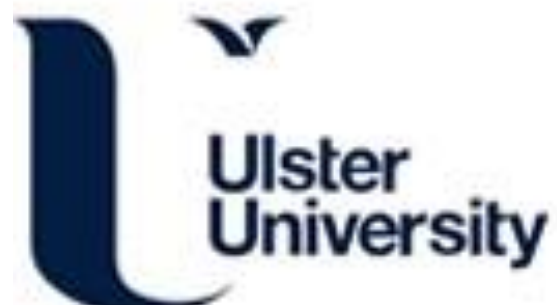

\section{Antenna Terminals based on Ultra-Compact Retrodirective Antenna Arrays}

Malyuskin, O., \& Fusco, V. (Accepted/In press). Antenna Terminals based on Ultra-Compact Retrodirective Antenna Arrays. IET Microwaves, Antennas and Propagation, na. https://doi.org/10.1049/iet-map.2016.0769

Link to publication record in Ulster University Research Portal

\section{Published in:}

IET Microwaves, Antennas and Propagation

\section{Publication Status:}

Accepted/In press: 24/08/2017

DOI:

10.1049/iet-map.2016.0769

\section{Document Version}

Author Accepted version

\section{General rights}

Copyright for the publications made accessible via Ulster University's Research Portal is retained by the author(s) and / or other copyright owners and it is a condition of accessing these publications that users recognise and abide by the legal requirements associated with these rights.

\section{Take down policy}

The Research Portal is Ulster University's institutional repository that provides access to Ulster's research outputs. Every effort has been made to ensure that content in the Research Portal does not infringe any person's rights, or applicable UK laws. If you discover content in the Research Portal that you believe breaches copyright or violates any law, please contact pure-support@ulster.ac.uk. 


\section{Antenna Terminals based on Ultra-Compact Retrodirective Antenna Arrays}

\begin{tabular}{|r|l|}
\hline Journal: & IET Microwaves, Antennas \& Propagation \\
\hline Manuscript ID & MAP-2016-0769 \\
\hline Manuscript Type: & Research Paper \\
\hline Date Submitted by the Author: & $13-$ Sep-2016 \\
\hline Keyword: List of Authors: & $\begin{array}{l}\text { Malyuskin, Oleksandr; ECIT, Queen's University Belfast } \\
\text { Fusco, Vicent; Queens University of Belfast, Ireland }\end{array}$ \\
\hline & $\begin{array}{l}\text { ANTENNA ARRAYS, ANTENNA DESIGN, ANTENNA PHASED ARRAYS, } \\
\text { ANTENNAS, ANTENNA RADIATION PATTERNS, ANTENNA THEORY, } \\
\text { DIFFRACTION, ELECTROMAGNETIC WAVE SCATTERING, PHASE CONTROL, } \\
\text { SCATTERING }\end{array}$ \\
\hline &
\end{tabular}




\title{
Antenna Terminals based on Ultra-Compact Retrodirective Antenna Arrays
}

\author{
Oleksandr Malyuskin, Vincent Fusco
}

The ECIT Institute, Queen's University Belfast, Belfast, BT3 9DT, UK

e-mail: smalyuskin@gmail.com

Abstract: A novel physical effect of angular super-resolution based on the Direction-of-Arrival (DoA) information sampling using highly oscillatory resonant evanescent harmonics generated in a dense retrodirective antenna array environment by an incident electromagnetic (EM) signal is demonstrated and analyzed using first principles, full-wave electromagnetic (EM) simulations and experimental study. This phenomenon enables realization of novel, ultra-compact antenna terminals with superdirective radiation patterns and spatial auto-focusing characteristics for any direction of arrival of the excitation waveform. The use of these antenna terminals should enhance the signal-to-noise ratio at the receiver, increase overall energy efficiency of the wireless communication or power transmission systems and lead to substantially lower interference and EM pollution across the wireless network area. Special attention is given to the antenna array operating in the presence of a flat metal ground plane or a ground plane with EM band-gap structure. Near-field scattering phenomena arising as a result of the antenna array coupling to the ground planes of both types and their effect on the far field radiation patterns are analyzed in detail. The results of the study should be important for the development of the antenna terminals to be employed in the energy-efficient wireless communication systems including the Internet of Things, wireless sensor networks and wireless powering systems.

\section{Introduction}

Wireless communications are employed extensively in modern electronic systems and devices with applications ranging from electronic healthcare [1] and industrial sensor networks [2] to smart homes [3] and driverless cars [4]. The classical area of radio frequency identification (RFID) has evolved into the Internet-of-Things concept [5] which refers to a communication network of different kinds of devices operating as an information-connected entity. It is estimated that by 2020 the IoT will include 15 billion to 50 billion connected devices across all sectors like transportation, industry, healthcare, smart cities, etc. [6]. While some of the devices will use wired connections the vast majority of the IoT components will be wirelessly connected. This presumes higher density of the wireless communication nodes per unit space within the wireless network area which will eventually lead to a high level of interference between the communicating devices and substantial electromagnetic (EM) pollution. Another problem relates to powering of a large number of wireless sensors and electronic tags - the use of batteries in a large volume of small electronic devices is prohibitive due to the need of their replacement and recycling and environmental 
compatibility. Wireless power transmission (WPT) using radiofrequency (RF) or microwave signal can provide an alternative way of powering batteryless sensors however it requires sufficient levels of the received EM power to overcome low efficiency of the existing RF rectifiers [7].

A novel class of smart antenna terminals (ATs) that could facilitate energy-efficient wireless data transmission and powering of batteryless sensors and lead to reduced level of EM pollution is proposed in the present paper. The EM operation of these ATs is grounded on the EM signal phase conjugation [8] which results in EM radiation auto-focusing between the communicating devices [9].

An AT is a key part of any wireless device enabling transmission and reception of EM signals carrying information [10]. Commonly, ATs radiate omnidirectionally for full spatial coverage in transmit (TX) and receive (RX) modes. Omnidirectional coverage in RX mode is crucial in simple, non-steered transceivers to ensure signal reception from an arbitrary DoA. At the same time in the TX mode generation of a narrow beam directed towards the intended communicator brings a number of benefits, including higher signal-to interference noise at the receiver for the same level of input power, interference reduction for the adjacent users in the wireless network, lower EM pollution and lower bit-error rate of a signal. These characteristics enable more energy-efficient communication or WPT systems [11] than is possible with the ATs radiating omnidirectionally.

One of the key requirements on the ATs is their small electrical size, with characteristic aperture dimension being smaller that the radiation wavelength. Existing compact ATs [13] are represented by the Yagi-Uda antennas, superdirective antenna arrays [13], [14] and the ESPAR (electronically steerable passive array radiator) antennas [15]. Superdirective array antennas [13], [14], [16] are of interest since they enable high radiation gain in a fixed direction in space by sacrificing omnidirectional signal coverage. The ESPAR antennas [15], [17] allow full azimuthal coverage by fixed electronic beam steering within azimuthal segments. However steering relies on the DoA estimation using power-hungry digital signal processing.

A new class of small ATs - electrically compact retrodirective arrays with auto-pointing and angular super-resolution radiation patterns is presented in this work. The unique properties of the proposed antenna systems are enabled by phase conjugation [8], [9], [18] of the EM signal across the AT ports.

The novel feature of this new class of retrodirective arrays is its electrically small size (not exceeding half-wavelength of radiation) combined with its superdirective far field pattern forming capability. The term 'superdirective pattern' means here a radiation pattern with beamwidth less than the Raleigh angular resolution limit [19]

$$
\theta_{A} \approx \lambda / L_{\mathrm{A}}
$$

In (19) $\theta_{A}$ is angular resolution in radians, $\lambda$ is a wavelength of radiation and $L_{A}$ is the antenna array characteristic (or maximum) dimension.

Phase conjugation (PC) of the incoming EM wavefront at the antenna array aperture forms the basis of retrodirective antenna array operation [8], [9], [18]. Retrodirective arrays automatically re-radiate an EM beam in the direction of the original source without any a priori knowledge of its angular position. Such arrays are built by merging dedicated circuitry performing PC with standard antenna arrays [20]. The inter-element spacings between the antenna elements in the conventional narrowband arrays are around $\lambda / 2$. These spacings enable mitigation of EM coupling effects that normally degrade dense antenna array performance due to lowering of the radiation resistance [13], [20]. In dense arrays (with reduced inter-element spacings) the scattered EM has a dominant reactive part which in general leads to higher EM coupling and antenna array performance degradation in terms of available return loss bandwidth [13], [20], [21]. 
In the present work we study the possibility of using resonant high intensity reactive near field generated in the antenna array environment in order to create electrically small (in the sense $L_{A}<\lambda / 2$ ) retrodirective ATs with auto-pointing and angular superresolution radiation patterns. Angular super-resolution is achieved by encoding/sampling the DoA information over the highly oscillatory evanescent harmonics generated in the dense antenna array environment [18]. As demonstrated in [18] the spatial sampling rate of the DoA information appears to be much higher than the characteristic spatial variation of the incident waveform (given by half-wavelength of radiation). Next, retrodirection of both the propagating and reactive parts of the EM field in the antenna array environment generates an array excitation vector [18] resulting in the radiated beam with auto-pointing properties.

The theory of the ultra-compact retro-directive antenna arrays operation in free space has been developed in [18] and its key points are summarized here as a starting point in Section II. In many practical scenarios antenna arrays operate in the presence of a ground plane or other complex media. The presence of EM scattering emanating from a ground plane leads to new features of the small retrodirective array operation discussed in detail in the present paper. Additionally, the ground plane is very often required for placement of electronic components or antenna array mounting. Performance of a small antenna array backed by a flat conventional ground plane and an electromagnetic bandgap (EBG) ground plane is studied with the aim to improve the radiation characteristics and antenna element return loss matching (Section III). Finally, in Section IV the examples of compact retrodirective antenna terminals applications in free-space communication scenarios are provided to demonstrate their superiority of proposed terminals over the standard antennas with fixed radiation pattern.

\section{Principle of Operation}

An example of a dense retrodirective $N$-element antenna array with inter-element spacings substantially less than the halfwave of radiation is shown in Fig.1(a). The array is illuminated by an EM plane wave (PW) with monochromatic time dependence $\exp (-i \omega t)$, suppressed in the subsequent analysis.

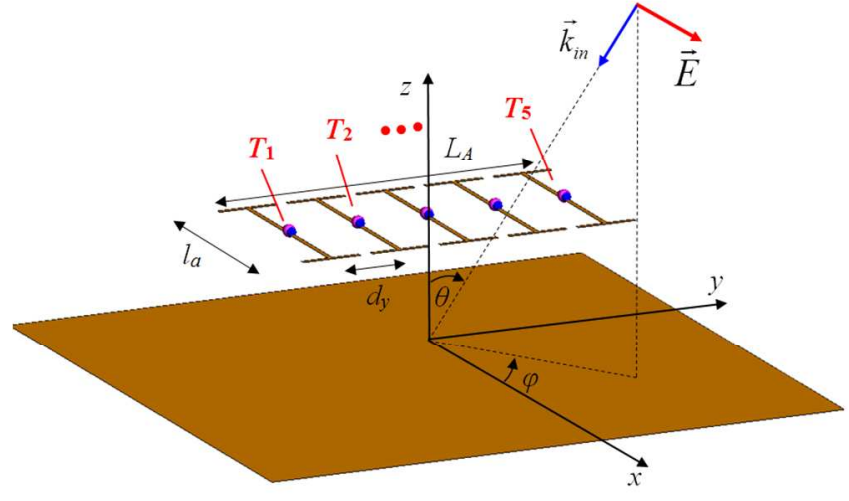

(a)

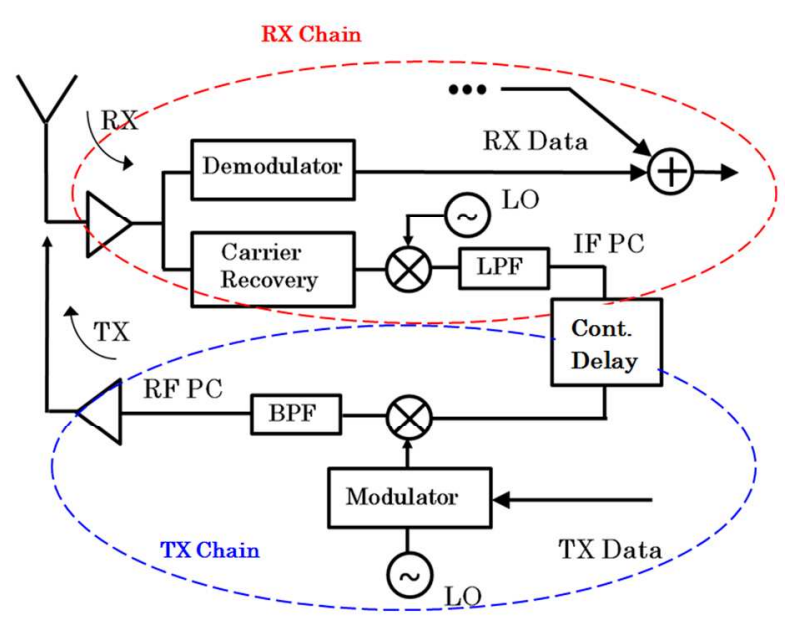

(b)

Fig.1. (a) A simulation model of an ultra-compact retrodirective antenna array illuminated by a linearly x-polarized monochromatic $P W$. The antenna array characteristic size $L_{a} \sim N d_{y} \sim 0.5 \lambda, N$ is a number of antenna elements, the inter-element spacings $d_{y}$ are of order of $0.1 \lambda . T_{n}, n=1,2, \ldots, N$ are the PC antenna ports. The antennas are end-loaded to ensure electric 
current resonance [13] and a perfectly conductive ground plane of finite size $\lambda x \lambda$ is used. The length $l_{a}$ of antenna elements satisfies $k_{0} l_{a} \approx 1$, with free space wavenumber $k_{0}=2 \pi / \lambda$.(b) Schematic of PC full-duplex communication circuitry enabling the array retrodirective operation [18], [22], [23].

The antenna array retrodirectivity is enabled by the PC architecture schematically shown in Fig.1(b), and PC operation discussed in detail in [18], [22], [23]. The array compactness means that its dimension $L_{a}$ is less than a half of the radiation wavelength, $L_{a} \leq 0.5 \lambda$. The arrays are formed by electrically compact resonance antennas [13], [14], with maximum size $l_{a}$ meeting the requirement $k_{0} l_{a} \approx 1$. In the simulation models the array elements are fed by the point-like idealized ports [24]. In practice the coaxial cable feeding is normally used [25] which in general do not affect the antenna array performance since the currents on the antenna elements are orthogonal to the feeding lines surface.

\subsection{AT in the $R X$ mode}

The total EM field $\boldsymbol{E}(\boldsymbol{r})$ in the antenna array environment is represented as a sum of an incident EM plane wave $\boldsymbol{E}_{i n}(\boldsymbol{r})=\boldsymbol{x}_{0} \exp \left(i \boldsymbol{k}_{i n} \cdot \boldsymbol{r}\right)$ and the scattered field $\boldsymbol{E}_{s c}(\boldsymbol{r})$ generated by the antenna electric currents $\boldsymbol{J}_{n}\left(\boldsymbol{r}^{\prime}\right)$

$$
\boldsymbol{E}(\vec{r})=\boldsymbol{E}_{i n}(\vec{r})+\boldsymbol{E}_{s c}(\vec{r})=\boldsymbol{E}_{i n}(\boldsymbol{r})+i \omega \mu_{0} \sum_{n=1}^{N} \int \hat{\boldsymbol{G}}\left(\boldsymbol{r}, \boldsymbol{r}^{\prime}\right) \boldsymbol{J}_{n}\left(\boldsymbol{r}^{\prime}\right) d \boldsymbol{r}^{\prime}
$$

where $\boldsymbol{k}_{i n}$ is the incident PW wavevector, $\hat{\boldsymbol{G}}$ is a dyadic Greens function of the array host medium [26]. In EM resonance [13], [25] high current densities $\boldsymbol{J}_{n}$ supported by the antenna elements result in high magnitude of the reactive scattered field which can significantly exceed the magnitude of the incident field, $E_{s c}(\boldsymbol{r}) \gg>E_{i n}(\boldsymbol{r})$. Following the analysis in [18] the scattered field in the array environment is expanded into a set of propagating and evanescent PWs [18], [27],

$$
\begin{aligned}
& \boldsymbol{E}_{s c}(\boldsymbol{r}) \sim \\
& \sum_{n=1}^{N} P_{n} \int_{-\infty}^{\infty} E(\boldsymbol{k}) \exp \left[i \chi \cdot\left(\boldsymbol{\rho}-\boldsymbol{\rho}_{n}\right)\right] \exp \left( \pm i k_{z}\left(z-h_{A}\right)\right) d \chi
\end{aligned}
$$

In (3) the DoA phase $P_{n}=\exp \left(i \boldsymbol{k}_{i n} \cdot \boldsymbol{\rho}_{n}\right)$ and the spectral amplitudes $E(\boldsymbol{k})$ are determined by the geometry and electric current amplitudes [18]. In (3) $\boldsymbol{\rho}$ and $\boldsymbol{\rho}_{n}$ are the observation point radius-vector in the array plane at $z=h_{A}$ and the radius-vector of the $n$ th antenna element center respectively. In (3) the "+" sign should be chosen for $z \geq h_{A}$ and the "-" sign should be taken for $z \leq h_{A}$, $h_{A}$ is the distance between the array plane and the reflector at $z=0$. It should be stressed that the wavenumber $\chi$ of the evanescent harmonics exceeds the wavenumber in free space $\chi>2 \pi / \lambda,[18],[27]$ and therefore when the evanescent components (reactive field) dominate over the incident field the DoA information $\Phi_{n}$ is encoded into the scattered field with higher spatial rate $\sim 2 \pi / \chi$ than the spatial rate of the incident wave in the free space. This leads to the effective increase of the array aperture and angular super-resolution of the retrodirected beam [18]. 


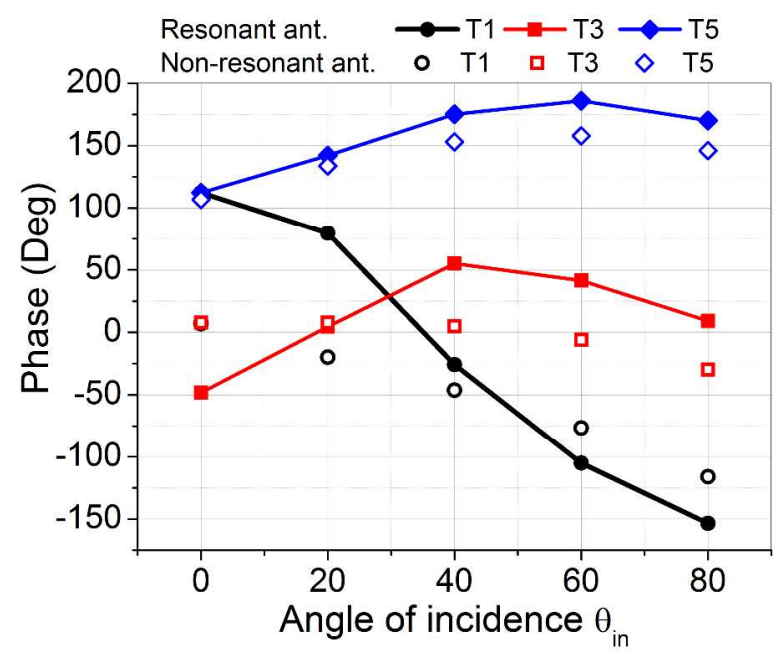

Fig.2. Simulated phase of the induced voltage across the ports $T_{1}, T_{3}, T_{5}$ of the resonance antenna array, solid dotted lines and across the non-resonant straight antenna receivers located in the same positions as the resonant antenna elements, hollow dots. Both arrays are in the receive mode. Simulation results are obtained using FEKO solver [22].

To illustrate the effect of higher spatial rate oscillations in the scattered near field we calculate the phase of the RX voltages across the antenna ports $T_{n}, n=1, . ., 5$, of resonant antennas in Fig.1(a) and compare the results with the voltages across the ports of non-resonant small antenna receivers $\left(l_{\mathrm{a}}=0.05 \lambda\right.$, no top-loading) located in the same positions as the resonant antenna elements above the reflector. The results are presented in Fig. 2 for the case of 5-element antenna array with inter-element separation $d_{y}=0.12 \lambda, h_{A}=0.25 \lambda$ and reflector size $1.0 \lambda \times 1.0 \lambda, \lambda=0.125 \mathrm{~m}$. These results are obtained with full wave EM solver FEKO [24]. The induced maximum current density across the resonant antennas is $10^{3}$ times higher than for the non-resonant antenna element case which leads to the dominance of the reactive scattered field over the incident field $E_{s c}(\boldsymbol{r}) \gg E_{i n}(\boldsymbol{r})$ in the resonant antenna array environment. As a result, the EM induced/received antenna terminal voltages experience considerably larger phase variation with the incidence angle across the resonance antenna array as compared to an electrically short non-resonant array, Fig.2. Physically, significant phase variation with the incidence angle is a consequence of the higher spatial oscillation rate of the evanescent harmonics in the antenna array plane resulting in larger phase shifts $\sim \chi d_{y}$ between the antenna elements.

\subsection{AT in the $T X /$ retrodirective mode}

The retrodirective properties of the compact ATs are enabled by the PC circuitry whose operation is considered in detail in [18], [22], [23]. It is important to stress that due to a very close electrical proximity of the retrodirective antenna elements (with separation of order of $\lambda / 10$ ), the realization of full-duplex communication link at the same frequency/time is challenging due to a very low isolation between the receive and transmit (TX) chains and therefore time or frequency division between the downlink and uplink needs to be used. In the time division link scenario the intermediate frequency (IF) PC signal can be delayed by an analog or digital delay unit. The frequency division duplex link requires a frequency offset [22], [23] which is typically small (less than 2-4\%) due to the narrow-band operation characteristics of the electrically compact resonant antenna arrays [18]. The effect of the frequency offset on the compact antenna terminal radiation pattern is studied in the next Section.

It should be also noted that in the simulation models used in this paper, the effect of complex PC circuitry in Fig.1(b) is 
modelled in the TX (retrodirective) mode by numerical complex conjugation of the RX voltage across the $n$-th antenna port, [18], [22], [28],

$$
v_{n T X}=v_{n_{R X}}^{*}
$$

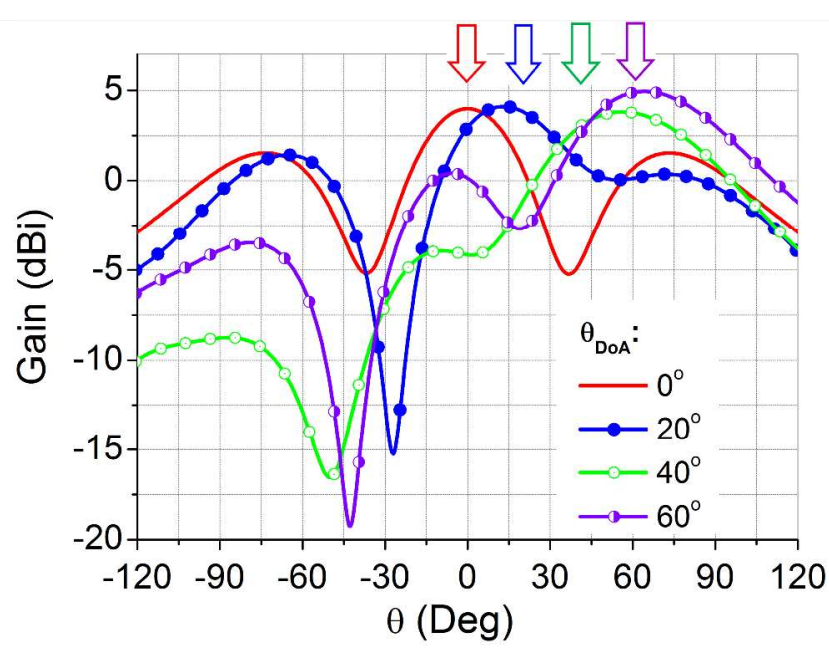

(a)

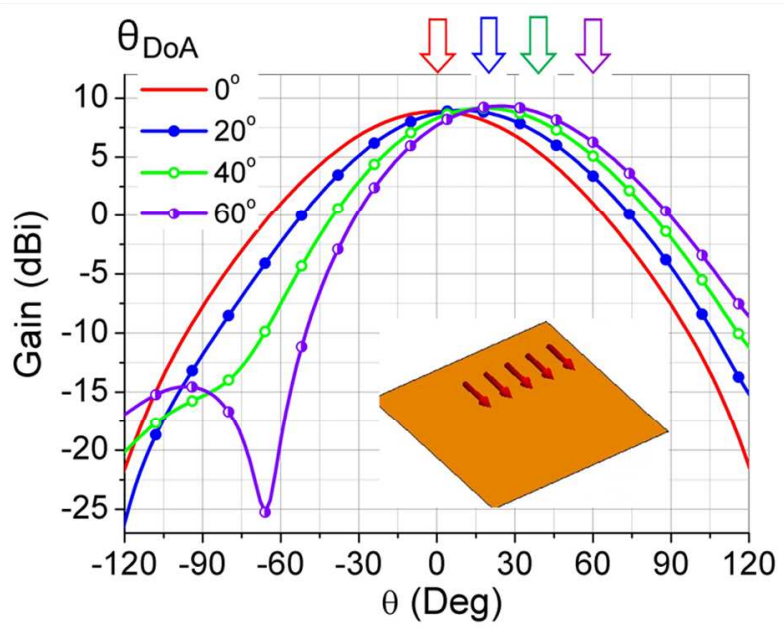

(b)

Fig.3. Simulated retrodirected radiation pattern at $2.4 \mathrm{GHz}$ in $y$-z plane generated by (a) resonance antenna array (a) and a counterpart array composed of Hertzian dipole sources located in the positions corresponding to the antennae centers, (b). The phase excitations of the Hertzian array are the conjugate of the incident field phase in the dipoles' positions. No scattering/EM coupling is present in the Hertzian dipole array case. Arrows on top of the graphs show the DoA. The simulated return loss fractional bandwidth of the resonance antenna elements is $\sim 3 \%$ at $-10 \mathrm{~dB}$ level, array elements are center-loaded with $10 \Omega$ lumped loads [18].

Fig. 3a) shows the retrodirected beam in yz plane generated by the resonant 5-element antenna array, Fig.1a) with dimensions $L_{a} \approx 0.5 \lambda, l_{a}=0.3 \lambda, d_{y}=0.12 \lambda, h_{A}=0.25 \lambda$, reflector dimensions $1.0 \lambda \times 1.0 \lambda, \lambda=0.125 \mathrm{~m}$ and Fig. $3 \mathrm{~b}$ ) shows the retrodirected pattern generated by a counterpart Hertzian point-like dipole array without scattering and EM coupling between the elements. The radiation patterns in Fig.3b) are computed using FEKO solver and they are the same as the classical pattern predicted by the array factor calculations [26]. It can be seen that the classical radiation pattern retro-directivity is limited to the small angular range near the boresight direction and angular bandwidth for the low angles of incidence is almost two times larger than the angular bandwidth of the patterns generated by the resonance antenna array. For more detail the properties of radiation patterns generated by the resonance and Hertzian arrays are summarized in Table I.

In this Table $\theta_{D o A}$ is the azimuthal angle of PW incidence in the yz plane, $\theta_{\max }$ is the elevation angle that corresponds to the maximum of the radiation pattern, $\Delta \theta_{3 \mathrm{~dB}}$ - is the angular beamwidth in the $y z$ plane at $3 \mathrm{~dB}$ level, and $G_{\max }$ is a maximum gain at $\theta_{\max }$. It can be seen that the angular beamwidth of the pattern radiated by a resonance array is almost two times smaller for small DoA angles. Another important feature in Table I is that the Hertzian array cannot retro-direct the beam in the DoA for oblique angles of incidence. At the same time the beam squint error, $\theta_{\max }-\theta_{D o A}$ for the resonance array is relatively moderate, $\sim 10^{\circ}$ and is only substantial for specific angle of incidence $\theta_{D o A}=40^{\circ}$. At the same time there are several deficiencies present in the radiation 
pattern of the resonance retrodirective array, particularly large level of sidelobes for $\theta_{D o A}=0^{\circ}$, large pointing error for $\theta_{D o A}=40^{\circ}$, these features are explained below.

Table 1. Properties of the Retrodirected Radiation Pattern

\begin{tabular}{|c|c|c|c|c|c|c|}
\hline$\theta_{D o A}$, & $\theta_{\max }^{\mathrm{res}}, \circ$ & $\theta_{\max }^{\mathrm{Hertz}}, \circ$ & $\Delta \theta_{3 \mathrm{~dB}}^{\mathrm{res}}, \circ$ & $\Delta \theta_{3 \mathrm{~dB}}^{\mathrm{Hertz}}, \circ$ & $\begin{array}{c}G_{\max }^{\mathrm{res}}, \\
\mathrm{dBi}\end{array}$ & $\begin{array}{c}G_{\max }^{\mathrm{Hertz}} \\
\mathrm{dBi}\end{array}$ \\
\hline 0 & 0 & 0 & 36 & 74 & 4.0 & 8.9 \\
\hline 20 & 13 & 10 & 44 & 72 & 4.1 & 9.0 \\
\hline 40 & 55 & 18 & 59 & 68 & 3.8 & 9.1 \\
\hline 60 & 64 & 25 & 58 & 67 & 4.9 & 9.3 \\
\hline 80 & 70 & 29 & 59 & 66 & 4.2 & 9.4 \\
\hline
\end{tabular}

\section{Compact PC ATs Characteristics and Limitations}

In this Section we outline the limitations of compact retrodirective arrays and suggest the ways to overcome them. The performance of the compact retrodirective arrays crucially depends on their geometry and the presence of scattering media/objects, specifically a ground plane.

\subsection{Antenna array geometry}

It has been demonstrated in [8], [28] that in general the retrodirected wave generated by a PC antenna array in free space is accompanied by a negatively refracted wave which radiates the EM energy in the unwanted direction. The effect of the negatively refracted wave in the retrodirective antenna array with a ground plane is shown in Fig.4(a),(b) for a 5-element antenna array with inter-element separation $d_{y}=0.12 \lambda, h_{A}=0.25 \lambda$ and reflector size $1.0 \lambda \times 1.0 \lambda, \lambda=0.125 \mathrm{~m}$ and DoA $40^{\circ}$.

It can be seen from the ray picture in Fig. 4(b) that the negatively refracted (NR) wave acquires additional phase shift $\Delta \psi$ with respect to the retrodirected beam,

$$
\Delta \psi=\frac{2 \pi}{\lambda} \cdot \frac{2 h_{A}}{\cos \theta}-\pi
$$

where the last term $-\pi$ rad takes into account phase shift due to the reflection from the conductive surface. Therefore the retrodirected beam and the beam arising as a result of the negative refraction have relative phase shifted by 55 degrees for $h_{A}$ $=\lambda / 4$. The approximate calculation of the beam pointing error using Kirchhoff's principle gives an estimate of around $15^{\circ}$ which is in agreement with full-wave simulation data in Fig.3(a). The pointing error is less pronounced for the low angles of DoA since the NR wave is reflected by the array from the radiation into the upper half-space and for large oblique DoA angles since the NR wave is radiated in broadside direction with little reflection into the upper half-space.

The effect of negative refraction can be eliminated or reduced in non-planar array geometries (antenna elements whose centers are not located on the single line in the vertical plane yz) as explained in [18]. In this case spatial coherence occurs only for the retrodirected EM wave and the NR wave is reduced due to the destructive interference. Another opportunity to reduce the NR 
wave adverse effect on the radiation pattern is to reduce the vertical separation $h_{A}$ which eliminates the radiation of the NR wave into the upper half-space. This height reduction is generally not always possible, in the case of a flat ground plane, without adversely affecting antenna return loss matching [25].

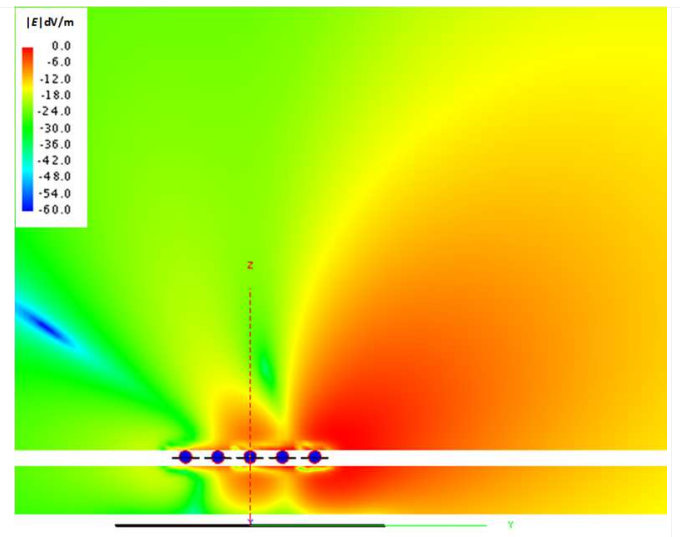

(a)

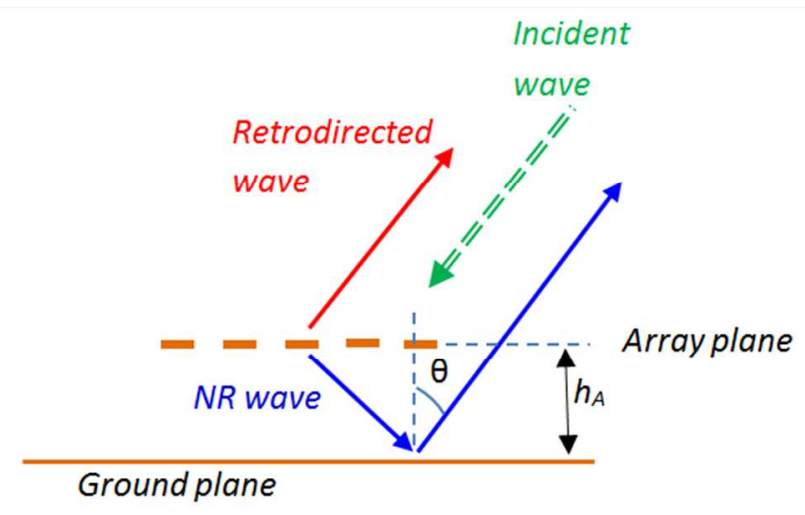

(b)

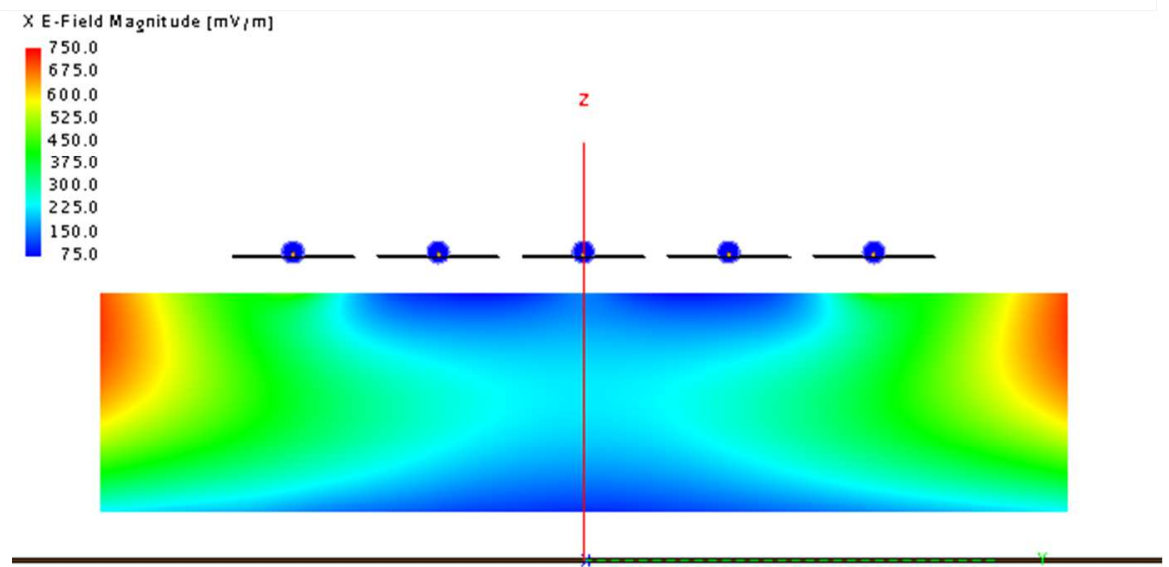

(c)

Fig.4. (a) Simulated retrodirected normalized E-field pattern at 2.4GHz in y-z plane generated by a resonance antenna array for the DoA 40 . (b) Ray representation of the propagating EM waves radiated by the antenna array. (c) Near E-field between the 5element antenna array and a flat metal ground plane with $\lambda / 4$ vertical offset distance, full-wave FEKO simulation. The antenna array in the receive mode, $D o A=0^{\circ}$.

\subsection{Ground plane effect}

A metal ground plane is often exploited to increase the antenna array gain in the boresight direction (along the $\mathrm{z}$ axis). However in the case of a compact retrodirective array large vertical offset $(\lambda / 4)$ required for antenna matching in the antenna system with a flat ground plane leads to a number of deficiencies including phase mismatch between the retrodirected and negatively refracted waves, Fig. 4 (a), (b) and also large edge field concentration, Fig.4(c) which results in significant side lobes in the retrodirected pattern, Fig.3(a), e.g. for $\mathrm{DoA}=0^{\circ}$. This $E$-field edge concentration generates substantially uneven amplitude distribution of the RX voltages across the antenna array ports, which in turn results in the generation of large side lobes in the 
retrodirected pattern, Fig.3(a), red line. The antenna array height above the ground plane can be reduced using antenna elements printed on dielectric substrate, however this does not eliminate the edge effects completely.

Another opportunity is to employ electromagnetic band-gap (EBG) ground plane [29], Fig.5(a) which allows much closer vertical separation between the antenna array and the ground plane. This enables much more uniform voltage distribution across the antenna array aperture which reduces the side lobes level. Also the NR wave effect is substantially reduced which leads to almost zero degree pointing error, Fig.5(b),(c). It can be seen in Fig.5(b),(c) that the front-to-back ratio is more than $7 \mathrm{dBi}$ for any DoA. For larger DoA angles (more than $40^{\circ}$ ) the pattern is widened in the yz plane which is caused by a close proximity ground plane that tends to direct the array radiation in the boresight direction. Nevertheless the retrodirectivity is preserved for an arbitrary DoA even though the antenna array is very compact.

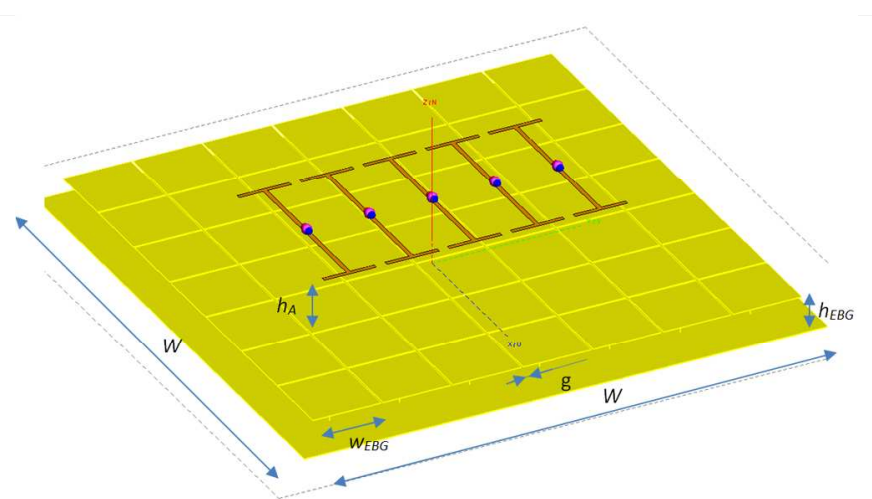

(a)

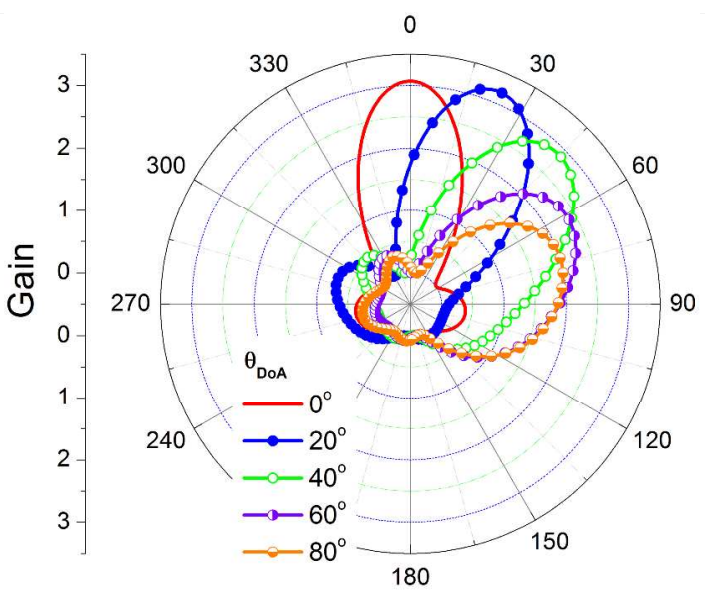

(b)

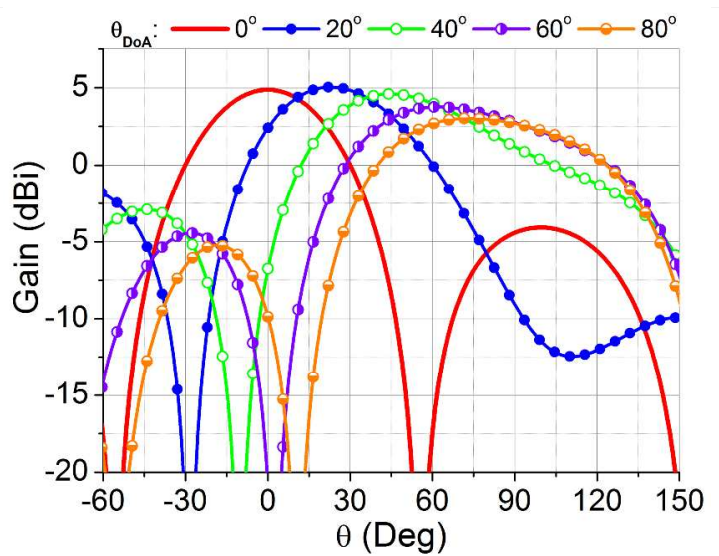

(c)

Fig.5.(a) 5-element planar antenna array with the EBG ground plane. The dimensions of the antenna array are the same as in Fig. 3(a): $l_{a}=0.3 \lambda, d_{y}=0.12 \lambda$, The ground plane parameters are $W=1.0 \lambda$, the square patch mushroom type EBG has dimensions $w_{E B G}=0.13 \lambda, h_{E B G}=0.05 \lambda, g=0.004 \lambda$, and $h_{A}=0.125 \lambda, \lambda=0.125 \mathrm{~m}$; (b) Simulated polar plot of the retrodirective patterns in the $y z$ plane (in terms of absolute gain) for various DoA; (c) Retrodirective patterns logarithmic gain in the angular range close to the radiation pattern maximum. The simulated return loss fractional bandwidth of the resonance antenna elements is $\sim 4.5 \%$ at -

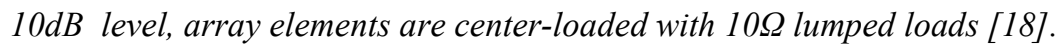




\subsection{Antenna array non-planar geometry and array miniaturization}

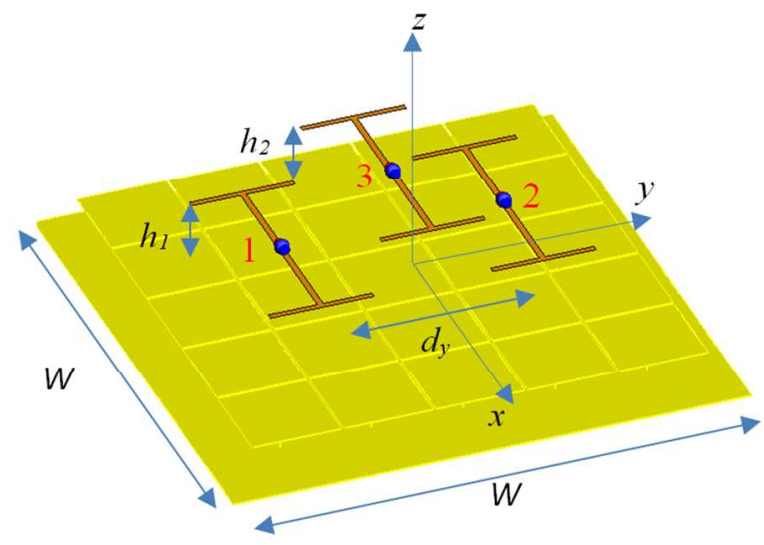

(a)

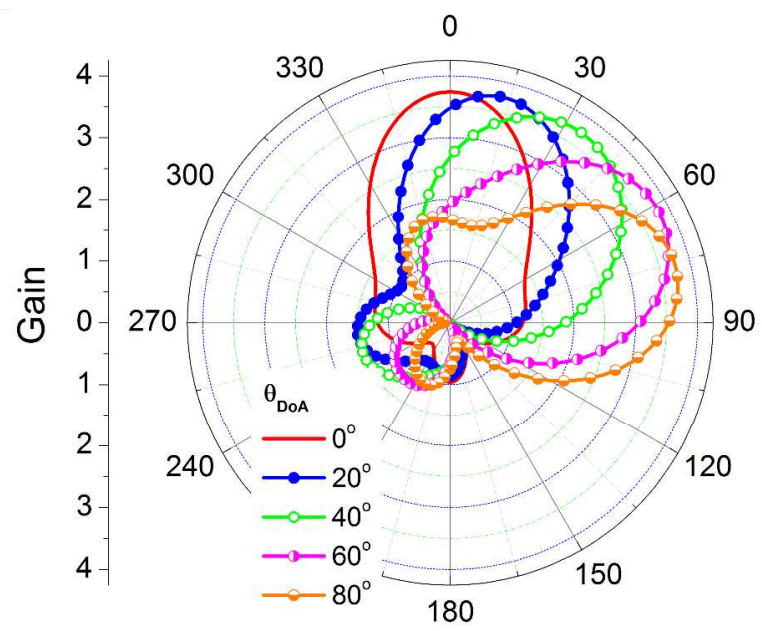

(c)

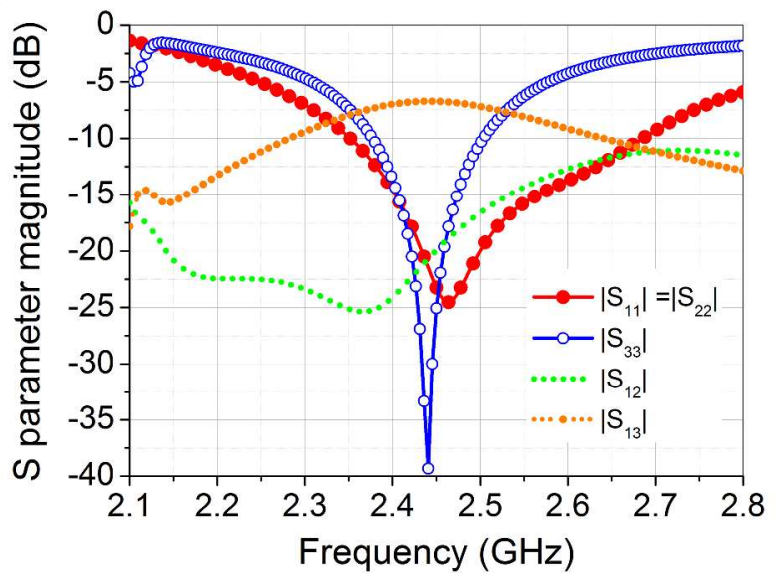

(b)

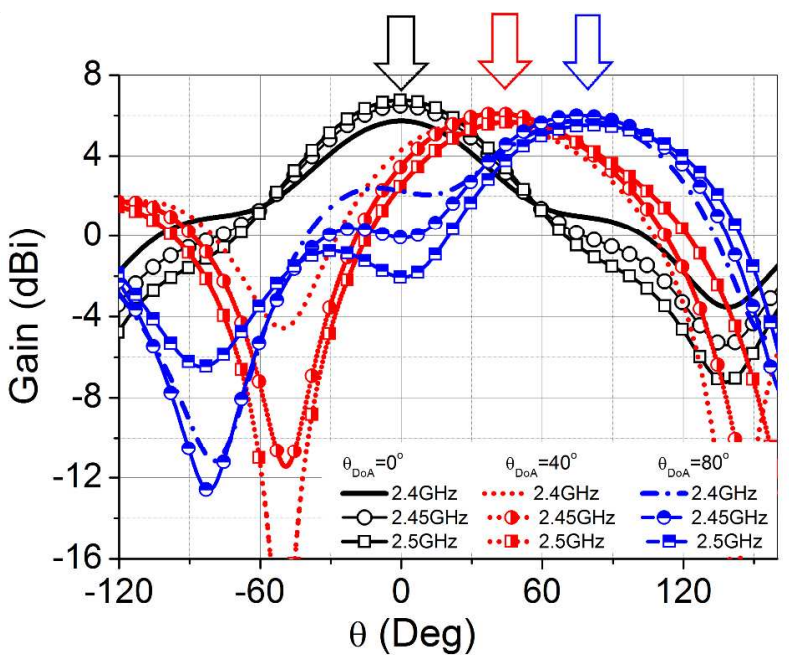

(d)

Fig.6.(a) 3-element non-planar (non-collinear in the yz plane) antenna array with the EBG ground plane. The dimensions of the antenna array: $l_{a}=0.3 \lambda, d_{y}=0.14 \lambda, h_{1}=0.125 \lambda, h_{2}=0.08 \lambda$ and the ground plane size $W=0.7 \lambda$, the antennae are center-loaded with $5 \Omega$ resistive load. The ground plane parameters are the same as for Fig. $5, w_{E B G}=0.13 \lambda, h_{E B G}=0.05 \lambda, g=0.004 \lambda$. (b) $S$ parameters magnitude for the antenna ports, full-wave simulation. The ports have different input impedances: 60 $\Omega$ for ports 1 and 2 and $35 \Omega$ for port 3. (c) Polar plot of the retrodirective patterns in the yz plane (in terms of absolute gain) for various DoA. (d) Retrodirective radiation patterns of a 3-element array at 2.4, 2.45 and 2.5 GHz for DoA 0, 40 and 80 degrees. Arrows on top of graph show the DoA.

As demonstrated in [18] non-planar (with non-collinear antenna element centers) retrodirective array geometry allows reduction of the NR wave effect through the destructive interference of partial NR waves from antenna elements arranged in three-dimensional geometries. Non-planar geometries also allow for the array spatial footprint minimization, Fig. 6(a). 
Figs. 6(a) and 6(c) demonstrate respectively the geometry and retrodirective radiation patterns of the non-planar antenna arrays above the EBG ground plane. It can be seen that even though the number of elements is reduced in the 3-element nonplanar array and its overall size is 30\% less as compared to its 5-element counterpart in Fig. 5, the radiation gain of a 3element array is higher and is almost constant with the angle of arrival, Table II.

Table 2. Properties of the Retrodirected Pattern by 3-Element Array with EBG Groundplane

\begin{tabular}{|c|c|c|c|}
\hline$\theta_{D o A}{ }^{\circ}$ & $G_{\max }, \mathrm{dBi}$ & $\begin{array}{c}\Delta \theta_{3 \mathrm{~dB}}, \\
\mathrm{y}-\mathrm{z} \text { plane }\end{array}$ & $\begin{array}{c}\Delta \theta_{3 \mathrm{~dB}}, \\
\mathrm{x}-\mathrm{o} \text { plane }\end{array}$ \\
\hline 0 & 5.73 & 87 & 64 \\
\hline 20 & 5.74 & 89 & 64 \\
\hline 40 & 5.70 & 103 & 64 \\
\hline 60 & 5.82 & 109 & 58 \\
\hline 80 & 5.73 & 92 & 52 \\
\hline
\end{tabular}

Table II also shows that the radiation pattern angular width is below the angular resolution limit (1) in both x-z and y-z planes. At the same time the squint angle in the $y-z$ plane does not exceed 3 degrees.

Simulations show that the array antenna elements return loss can be readily matched in $4 \%$ to $10 \%$ fractional bandwidth at 10dB level, Fig.6b) without considerable resistive loading normally used in the electrically small receive antenna arrays [13]. Optimal matching bandwidth can be achieved by varying the input impedance of the relevant PC circuitry which takes into account the antenna array geometry. For example, in the 3-element array shown in the Fig.6(a) the optimal input impedances are found to be $60 \Omega$ for the elements 1 and 2 and $35 \Omega$ for the port 3 due to different near field coupling in the 3-element non-planar array with a EBG ground plane.

The results in this Section suggest that a non-planar 3-element array represents the most optimal ultracompact retrodirective antenna terminal in terms of the balance between the achievable radiation gain and the antenna system size and complexity.

\subsection{Frequency-Division Duplex Link Using a Retrodirective Terminal}

It has been pointed out in Section II B that due to the low RX-TX isolation, any duplex link involving compact retro-directive terminal needs to be based on the frequency or time division access. Here we study the effect of a small frequency offset on the retrodirective pattern of a 3-element array in Fig. 6a) as a representative example. For narrowband arrays, the frequency offset is normally small, up to $2 \%-5 \%$ [22] and cannot exceed the return loss bandwidth which is around 5\%, in the present example Fig.6b). Fig. 6(d) demonstrates the simulated retrodirective radiation patterns of a 3-element antenna array with geometry parameters described in Fig.6(a).

The excitation vector of the antenna array is generated by the incident wave at $2.4 \mathrm{GHz}$ and in the retrodirection mode only the frequency is changed in the $4 \%$ fractional bandwidth (the array excitation vector is the same for $2.4,2.45$ and $2.5 \mathrm{GHz}$ ). Simulations across the range of DoA show that in this narrow band the retrodirectivity is preserved with high accuracy, Fig.6(d). This property opens up a possibility for a full-duplex communication link using frequency-division multiple access with a pilot tone at only the downlink frequency. 


\section{Experimental verification}

A planar 3-element antenna array prototype with flat ground plane has been fabricated to validate some of the analytical and simulation results in Sections 2, 3. The geometry and respective simulation model are shown in Fig.7(a), the array prototype is shown in Fig.7(b). Capacitive dipole element coupling is used to excite the antenna elements and eliminate the need in complex balanced balun feeding. In the RX mode the array is illuminated in the yz plane by a linearly x-polarized plane wave. In the experimental setup the array is illuminated in the anechoic environment by a standard horn antenna located at $3.5 \mathrm{~m}$ distance from the retrodirective array plane. The experimental setup and measurement technique are described in detail in [30]. Fig.7(c) shows the measured and simulated return loss in the $2.1-2.5 \mathrm{GHz}$ frequency band.

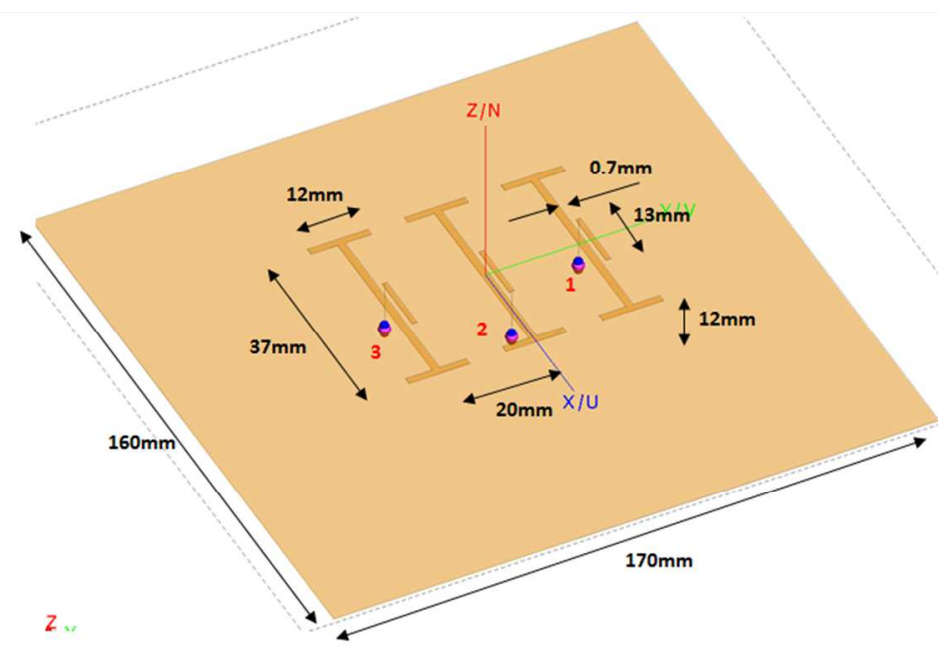

(a)

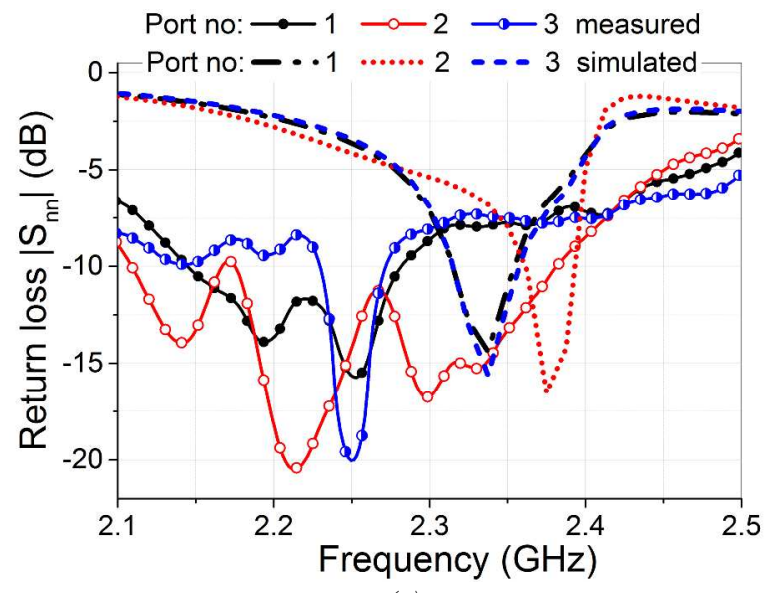

(c)

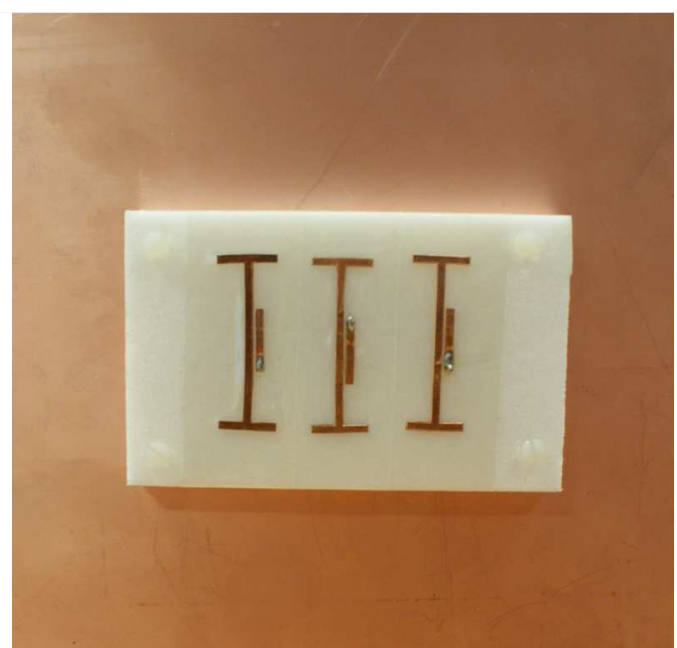

(b)

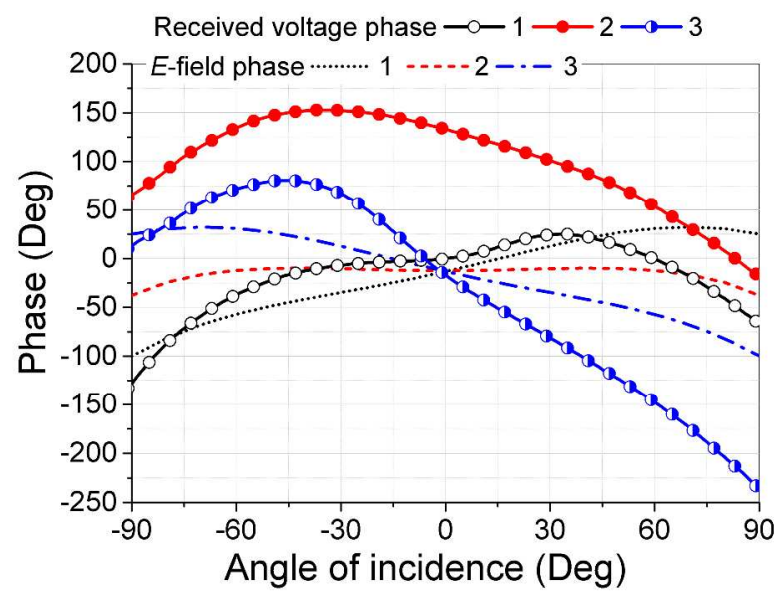

(d)

Fig.7. Planar 3-element antenna array FEKO simulation model(a) and fabricated prototype (b); simulated and measured antenna return loss (c) and measured $R X$ voltage across the ports $1-3$ at $2.25 \mathrm{GHz}$, (d). In (d) the simulated phase of the incident field in the locations corresponding to the antenna element centers is also shown for comparison.

It can be seen that simulation and measurement results are only in qualitative agreement due to substantially different feeding port structure in the FEKO simulation model (point-like feeding port) and experimental prototype (SMA bulk-head $50 \mathrm{Ohm}$ coaxial feeding port). It is interesting to note that the experimental data show almost two times wider return loss matching bandwidth as compared to the simulation-based prediction. It is believed that the resonance frequency shift in return loss, Fig.7(c) occurs due to presence of Rohacell dielectric [31] with relative permittivity 1.12, supporting the antenna elements and 
fabrication tolerance $(\sim 0.5 \mathrm{~mm})$. Next, Fig. 7 (d) shows the measured phase across the antenna element ports and simulated incident E-field phase in the locations corresponding to the antenna elements centers above the ground plane (with dipole antennas removed). It can be seen that the phase variation across the ports of the resonant antenna elements exceeds the phase variation of the incident wave by more than 100 degrees.

The measured and simulated retrodirective radiation patterns generated by a 3-element array are shown in Fig. 8 for the DoA 0, 30 and 45 degrees. It can be seen that due to the low elevation of the array above the ground plane (less than $0.1 \lambda$ at $2.25 \mathrm{GHz}$ ), each partial antenna element radiation pattern has a maximum at the boresight elevation angle $0^{\circ}$. Nevertheless the 3 -element array can efficiently steer the retrodirective beam in 0-45 degrees range. As was suggested in Section 3, the use of EBG ground plane and triangular antenna arrangements in the yz plane can substantially increase the beam steering range. Optimization of the retrodirective antenna array performance will be carried out in future work.

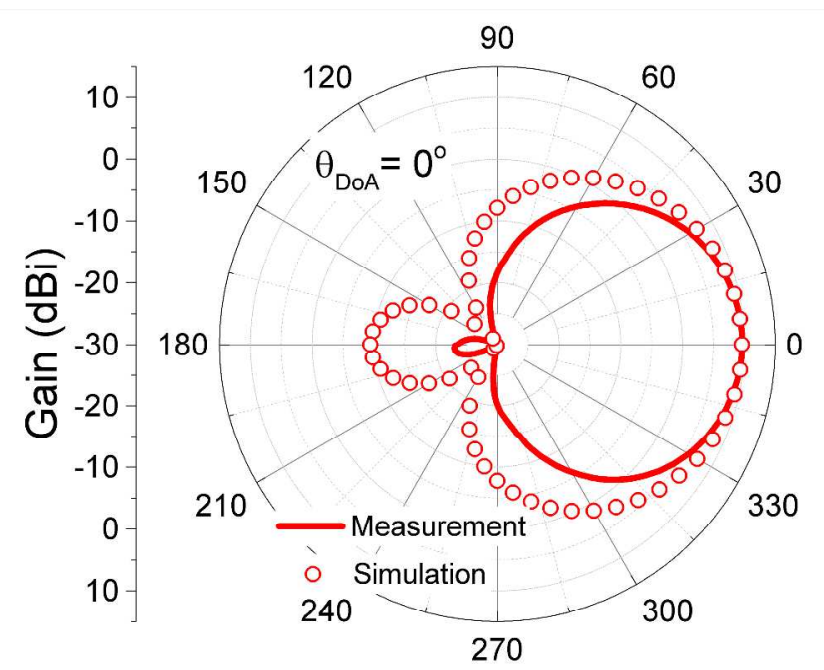

(a)

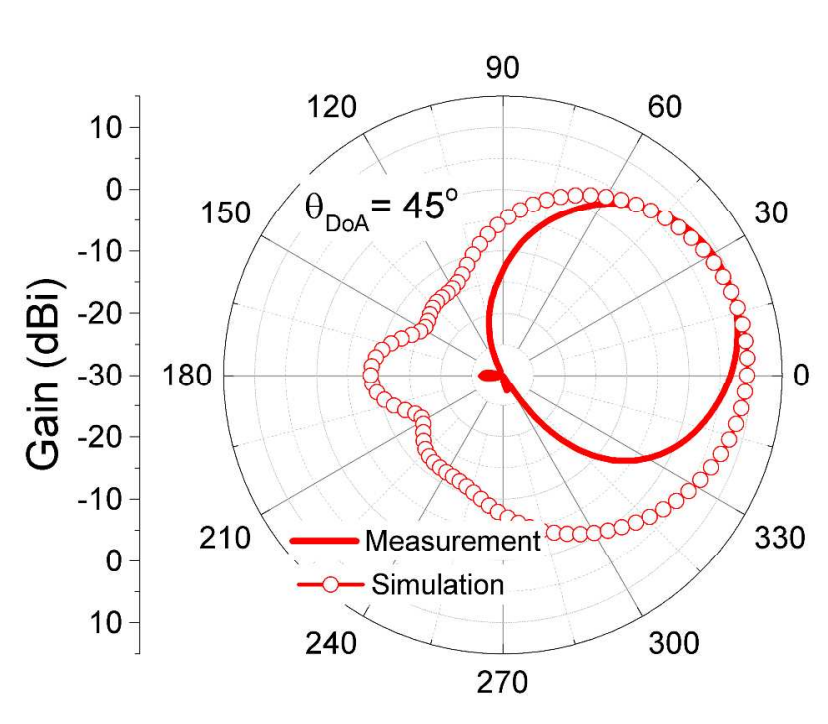

(c)

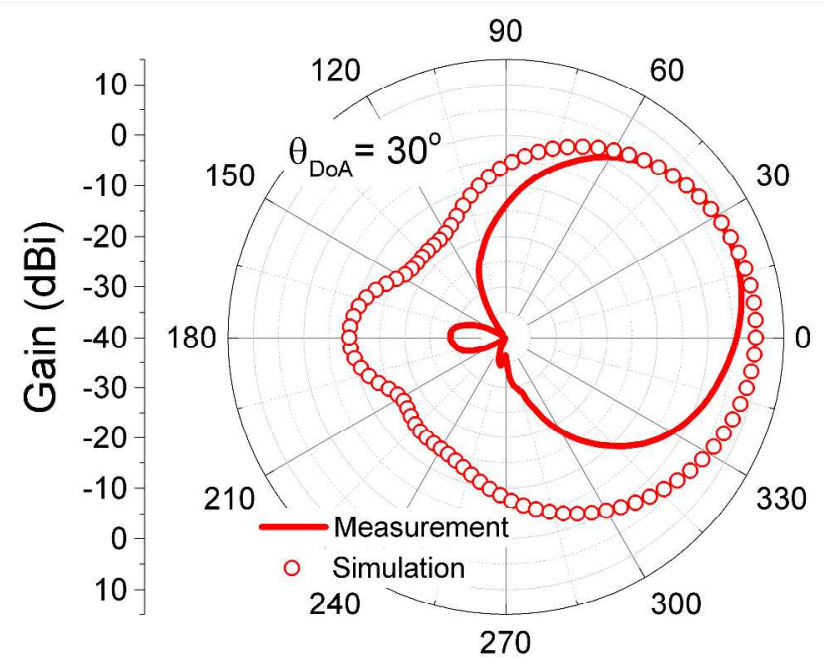

(b)

Fig.8.Retro-directive radiation patterns generated by a 3-element array at $2.25 \mathrm{GHz}$ for DoA (a)0, (b)30 and (c)45 degrees. Solid line measurements, dotted line - simulations 


\section{Conclusions}

A novel concept for electrically compact retrodirective antenna arrays with super-resolution radiation patterns and autopointing has been presented and the physical principles underlying its operation explained. It is demonstrated that close antenna spacings of order of tens of a wavelength allow the DoA information encoding/sampling with a high spatial rate exceeding the spatial rate of the incoming EM signal in free-space. The super-resolution properties of the retrodirective patterns generated by the proposed ATs are studied by means of full wave simulations and experimentally. It is particularly demonstrated that the RX/TX signal power that does not degrade with large angles of arrival like in the case of fixed-pattern antenna terminals with maximum radiation pattern in boresight direction.

Next, characteristic electromagnetic properties and limitations of ultra-compact antenna terminals are discussed using fullwave simulations and experimental work. Particularly we showed how the performance of compact antenna terminals can be improved using EBG ground planes and geometry of the antenna arrays.

The proposed antenna arrays can find applications in energy-efficient wireless communications with small spatial footprint of the transceivers. Also, the auto-pointing properties of small retrodirective antenna terminals should result in lower interference and the possibility of frequency reuse in ad-hoc wireless sensors networks.

\section{Acknowledgement}

The work has been sponsored by the Leverhulme Trust UK under the grant no RPG-2013-117.

\section{References}

[1] H. Alemdar and C. Ersoy, 'Wireless sensor networks for healthcare: A survey,' Computer Networks, vol. 54, no. 15, pp. 2688-2710, 2010.

[2] G. Zhao, 'Wireless Sensor Networks for Industrial Process Monitoring and Control: A Survey,' Network Protocols and Algorithms, vol. 3, no. 1, pp. 46-63, 2011.

[3] T. Mendes, R. Godina, E. Rodrigues et al, 'Smart Home Communication Technologies and Applications: Wireless Protocol Assessment for Home Area Network Resources', Energies, vol. 8, pp. 7279-7311, 2015. doi:10.3390/en8077279

[4] M. Harris, 'Google Wants Its Driverless Cars to Be Wireless Too,' IEEE Spectrum, 5 Feb 2016 [Online] http://spectrum.ieee.org/cars-thatthink/transportation/self-driving/google-wants-its-driverless-cars-to-be-wireless-too .

[5] V. Cerf, M. Senges, 'Taking the Internet to the Next Physical Level,' Computer, vol.49, no.2, pp.80-86, 2016.

[6] Internet of Things, Keysight technologies application notes [Online] www.keysight.com/find/IoT-Insight

[7] N. Carvalho, A. Georgiadis, A.Costanzo et. al, 'Wireless Power Transmission: R\&D Activities Within Europe', IEEE Trans Microwave Theory Techniques, vol. 62, no.4, pp. 1031-1045, 2014.

[8] O. Malyuskin, V. Fusco, A. Schuchinsky, 'Microwave phase conjugation using nonlinearly loaded wire arrays', IEEE Trans. Antennas Propagat., vol.54, no.1, pp. 192-203, 2006.

[9] K.M.K.H. Leong, Y. Wang, T. Itoh, 'A full duplex capable retrodirective array system for high-speed beam tracking and pointing applications', IEEE Trans. Microwave Theory Techniques, vol.52, no.5, pp. 1479-1489, 2004.

[10] S. Saunders, A. Aragón-Zavala, Antennas and Propagation for Wireless Communication Systems, Wiley 2007.

[11] A.J. Goldsmith, Wireless Communications, Cambridge University Press, 2005.

[12] Pak Chan, V. Fusco, '5.8 GHz full duplex amplitude shift keying retrodirective interrogator array', Microwave Optical Technology Letters, vol. 55, no.1, pp. 160-164, Jan. 2013.

[13] R.C. Hansen, R. E. Collin, Small Antenna Handbook, John Wiley \& Sons, Inc. 2011.

[14] K. Fujimoto, A. Henderson, K. Hirasawa, and J. R. James, Small Antennas, Wiley, 1987.

[15] H.-T. Liu, S. Gao, and T.-H. Loh, 'Electrically Small and Low Cost Smart Antenna for Wireless Communication', IEEE Trans. Antennas Propagat., vol.60, no.3, pp. 1540-1549, March 2012. 
[16] A. D. Yaghjian, T. H. O’Donnell, E. E. Altshuler, and S. R. Best, 'Electrically small supergain end-fire arrays', Radio Science, vol. 43, RS3002, pp1-13, 2008.

[17] Y. Zhou, R. S. Adve, Sean Victor Hum, 'Design and Evaluation of Pattern Reconfigurable Antennas for MIMO Applications', IEEE Trans. Antennas Propagat., vol.62, no.3, pp. 1084-1092, March 2014.

[18] O. Malyuskin, V. Fusco, 'Ultra-Compact Retrodirective Antenna Arrays with Superdirective Radiation Patterns', IEEE Trans. Antennas Propagation, no.7, vol.64, pp. 2923-2935, July 2016.

[19] R.C. Hansen, 'Fundamental limitations in antennas', Proceedings IEEE, vol. 69, no.2, pp. 170-182, Feb. 1981.

[20] R. Mailloux, Phased Array Antenna Handbook, 2nd ed. Norwood, MA, USA: Artech House Antenna Library, 2005.

[21] K. Buell, H. Mosallaei and K. Sarabandi, 'Metamaterial Insulator Enabled Superdirective Array’, IEEE Trans. Antennas Propagat., vol. 55, no.4, pp. 1074$1085,2007$.

[22] V. Fusco, N. Buchanan, 'Developments in retrodirective array technology', IET Microw. Antennas Propagat., vol. 7, no. 2, pp. 131-140, 2013.

[23] L. Chen, Y. C. Guo, X. W. Shi, and T. L. Zhang, 'Overview on the Phase Conjugation Techniques of the Retrodirective Array', Int. Journal Antennas Propagat., vol. 2010, pp.1-10, Article ID 564357.

[24] FEKO - Ports, Sources, Frequency and Meshing, [Online] www.feko.info, http://www.antenna-theory.com/tutorial/feko/feko2.php

[25] Kraus, J. D., and R. J. Marhefka, Antennas, 3rd ed., McGraw-Hill, New York, 2001.

[26] J.A. Kong, Electromagnetic Wave Theory, EMW Publishing, 2000.

[27] T.B. Hansen, A.D. Yaghjian, Plane-Wave Theory of Time-Domain Fields: Near-Field Scanning Applications, Wiley IEEE, 1999.

[28] V. Fusco, O. Malyuskin, N. Buchanan, ‘Active phase conjugating lens with sub-wavelength resolution capability', IEEE Trans. Antennas Propagat., vol.58, no.3, pp. 798-808, 2010.

[29] F. Yang, Yahya Rahmat-Samii, Electromagnetic Band Gap Structures in Antenna Engineering, Cambridge University Press 2009.

[30] O. Malyuskin, V. Fusco, 'Experimental Study of Electrically Compact Retrodirective Monopole Antenna Arrays', submitted to IEEE Trans. Antennas Propagation, August 2016.

[31] Rohacell substrate datasheet, http://www.rohacell.com/sites/lists/PP-HP/Documents/ROHACELL-Dielectric-Properties-EN.pdf 